\title{
Antibacterial properties and mechanism of gold nanoparticles obtained from pergularia daemia leaf extract
}

\begin{abstract}
The development of environmental dealings for the green synthesis of metal nanoparticle using plant mediated process is evolving an important branch of nano biotechnology. It offers a controlled synthesis of metal nanoparticles with well-defined shape, size, composition and morphology. The present study reveals the green synthesis of gold nanoparticle that is nontoxic, novel and eco-friendly. This synthesis approach is cost effective, eco-friendly and promising method for generation of new materials which can be used for many potential applications in field of science and medical. The colour change in UV-visible spectroscopy at absorption peak of $536 \mathrm{~nm}$ occurred due to the surface Plasmon Resonance effect. The antimicrobial activity and mechanism of gold nanoparticles studies for the bacterial growth of gram-negative bacteria like E.coli, Pseudomonas aeruginosa and gram-positive bacteria like Bacillus subtilis respectively. Highly crystalline nanoparticles of size $10 \mathrm{~nm}$ are confirmed from HR-TEM which supports with d spacing of XRD analysis. Further the characteristics of the obtained gold nanoparticles were studied using XRD, UV-Vis spectroscopy, Scanning Electron Microscopic, and High Resolution Transmission Electron microscopy and the results are presented in detail.
\end{abstract}

Volume 6 Issue I - 2017

\author{
Senthilkumar S,' Kashinath L, ${ }^{2}$ Ashok M, ${ }^{3}$ \\ Rajendran $A^{\prime}$ \\ 'Department of Physics, Nehru Memorial College \\ (Autonomous), India \\ ${ }^{2}$ Center for Materials Science and Technology, University of \\ Mysore, India \\ ${ }^{3}$ Department of Physics, National Institute of Technology, India
}

Correspondence: Annamalai Rajendran, Department of Physics, Nehru Memorial College(Autonomous), Puthanampatti, Tiruchirappalli,Tamilnadu, 621007,Tel 919943320598:

Emailneelrajnmc@gmail.com

Received: August 04, 2017 | Published: August 29, 2017

Keywords: Pergularia daemia leaf, Antimicrobial activity

Abbreviations: ROS, Reactive Oxygen Species; HRTEM, High Resolution Turrning Electron Microscope

\section{Introduction}

Gold is generally used in the nitrate form to induce antimicrobial effect, but when gold nanoparticles are used, there is a huge increase in the surface area available for the microbial expose on gold ions are used in the formulation of dental resin composites, in coatings of medical devices, in air sanitizer sprays, wet wipes, socks, detergents, soaps, tooth pastes, pillows, washing machines. The green route method for synthesis and development for Gold nanoparticles (AuNPs) is evolving to an important branch of bio-nanotechnology.$^{1,2}$ There is no need to introduce and explain the physical and chemical properties of gold nanoparticles, sinceit has been used from ages ${ }^{3,4}$ However, nanotechnology is anticipated to open a novel opportunity to prevent and fight against the disease causing organism using atomic scale tailoring of materials.$^{5}$ In the field of nanotechnology, the synthesis of nanoparticles of different sizes, chemical compositions, and controlled morphology desirable for mono-dispersability is very essential because they exhibit unique properties which are not seen in bulk materials ${ }^{6-7}$ Recently, there is an urge to develop for friendly environmentally benign nanoparticle synthesis processes.$^{8}$ In recent years, the plantmediated synthesis of nanoparticle applicable on biological woks are more beneficial over physical and chemical methods because of its eco-friendly, cost-effective and replace the complex process of maintaining cell cultures and could be suitably scaled up for largescale nanoparticle synthesis method and with different parameters. 5 NPs have useful variation applications in, and coating formulations .9${ }^{10}$ Recently, research focused towards the development of biologically synthesized AuNPs.$^{11,12}$ because of its extensive applications in new technology in the field of electronics, material science and medicine at nanoscale.${ }^{13-14}$ The biosynthesis of AuNPs have been already reported from the leaf extract of Adeniumobesum.${ }^{15}$ Eucalyptus chapmanina.${ }^{16}$ Mangrove plants.$^{18}$ Lantana camera.${ }^{19}$ etc. Mainly, AuNPs were synthesized for medicinal applications like cancer treatment; bacterial and fungal tolerance towards metal and intracellular uptake of metals .Green synthesis of AuNPs by the extract of plant may increase their physiochemical properties at nanoscale and can act as materials for potential application in the field of medicine. In modern era; emergence of resistant microorganisms to multiple antimicrobial agents is a major threat to public health. Since from the ancient time, silver ions was used as potent antimicrobial agent and gold ions are widely used to kill bacteria in catheters burn wounds and dental works . ${ }^{19-20}$ In last few decades, the green nanoscience and nanotechnology has proven to offer and excel the opportunities for exploring the effect of metal nanoparticles for the antimicrobial activity and environmental issues.

\section{Experimental}

\section{Collection of plant material}

The fully matured fresh leaves of Pergularia daemia were collected from Nehru Memorial College Campus, India for the synthesis of AuNPs. It was authenticated by a plant taxonomist from the Department of Plant Science, Nehru Memorial College Puthanampatti. Gold (I) chloride, in this experiment were obtained from Sigma (Bangalore, India) and Merck (Mumbai, India) with high purity. Deionized water was used throughout the experiments.

\section{Biosynthesis of gold nanoparticles}

The collected fresh Pergularia daemialeaves were thoroughly washed with tap water and with double distilled water thrice remove dust particles. The small pieces of leaves were then shade dried, and finally used for the biosynthesis of AuNPs. Plant leaf extracts were prepared by boiling $10 \mathrm{~g}$ of dried leaves in $100 \mathrm{~mL}$ of deionized water in $300 \mathrm{~mL}$ Erlenmeyer flask for $30 \mathrm{~min}$ at $60^{\circ} \mathrm{C}$. Then the solution was filtered through a Whatman no.1 filter paper and kept the resultant product in stored at $20^{\circ} \mathrm{C}$ and use within $1 \mathrm{~h}$ Pergularia daemia leaf extracts at different time interval i.e. $(0,30,60,90$, and $120 \mathrm{~min})$ was 
treated with $100 \mathrm{~mL}$ of aqueous $1 \mathrm{mM} \mathrm{HAuCl} 4$ solution with constant stirring in Erlenmeyer flask for reduction of $\mathrm{Au}+$ ions and periodically color change of the solution was observed from yellow to wish red solution.

Pergularia daemia leaf extract without AuNPs did not show any change in colour (Figure 1). The colour of the extract changed to light brown within $10 \mathrm{~min}$, and later, it changed to dark brown during 15 min incubation period after which there was no significant change occurred.

\section{Characterizations of the synthesized gold nanoparticles}

The signature of AuNPs was monitored by UV-Visible spectroscopy, which is powerful tool for the characterization of colloidal particles. The synthesized pure AuNPs in presence of leaves extract was monitored by observing the UV-Vis analysis in the wavelength range 300 to $800 \mathrm{~nm}$. The UV-Vis spectra were recorded in a Shimadzu Kyoto, Japan) UV-Vis spectrophotometer operating at a resolution of $1 \mathrm{~nm}$. The structure and size of synthesized AuNPs was determined by X-ray diffraction spectroscopy. The air dried nanoparticles were coated onto XRD grid and analyzed for the formation of AuNPs by Philips X-Ray Diffractometer.XRD pattern was recorded using $\mathrm{CuK} \alpha$ radiation $\left(\lambda=1.54060 \mathrm{~A}^{\circ}\right)$ with nickel monochromatic in the range of $2 \Theta$ from $10^{\circ}$ to $80^{\circ}$. The average crystallite size of the synthesized AuNPs was calculated using Scherer's formula $D=(0.9 \lambda) / \beta \operatorname{Cos} \theta$. The morphological features of AuNPs were studied by field emission scanning electron microscope (FESEM) and high resolution transmission electron microscope (HRTEM) using JEOL-Model 6390 machine.

\section{Antimicrobial activity of Au Nanoparticles}

The antimicrobial activity of AuNPs determined using disc diffusion method. The antibacterial activities of gold nanoparticles were studied against the gram-negative and gram positive bacterial strains, staphylococcus aurous, pseudomonas aeruginosa and Escherichia Coli. The bacterial strains were grown in nutrient broth at $37 \circ^{\circ} \mathrm{C}$ with continuous shaking at $200 \mathrm{rpm}$ for $24 \mathrm{~h}$. The nanoparticles were sub-cultured on Muller Hinton agar medium (MHA) using standard method and incubation was kept for a period $48 \mathrm{~h}$ at $50^{\circ} \mathrm{C}$ and stored in $5^{\circ} \mathrm{C}$ in refrigerator to maintain stock culture. The serial dilutions were conducted with different level of concentrations $100 \mu \mathrm{L}, 200$ $\mu \mathrm{L}$, and $300 \mu \mathrm{L}$ of gold nanoparticles. The zone growth of the plate incubation was observed after $48 \mathrm{~h}$ using a negative bacterium as a standard positive control. Growth kinetics was determined by measuring optical density at $560 \mathrm{~nm}$ at every $1 \mathrm{~h}$ interval from the time of incubation.

\section{Results and discussion}

In the emerging field of nanotechnology, the formation of nanoparticles using biological materials as reducing agents is receiving more attention, especially in the last decade. Successful biosynthesis of AuNPs by the filtrate of Pergularia daemia leaf was confirmed by the change in the color of the filtrate from yellow to dark brown after the addition of AuNPs but control flasks, containing only the AuNPs solution (without plant filtrates) did not show any color change. AuNPs have free electrons which have risen to SPR absorption due to the vibration of electrons of the metal nanoparticles in resonance with light wave and it is an indication of formation of AuNPs.

Figure 1 shows the UV-Visible spectrum of gold nanoparticles recorded from the reaction medium at the interval of $(0,30,60,90$ and
120 mins). The maximum absorption characteristic Surface Plasmon peak is located at $536 \mathrm{~nm}$ indicates the formation and presence of Au nanoparticles. The color changes are due to excitation of surface Plasmon vibration in gold nanoparticles. The SPR and color change in AuNPs particles. The maximum absorption spectra band are shifted to longer wavelength are due to the increase in concentration level and time of exposure on surface of Au nano particles which indicates the formation of more or larger number Au particles in different size from $536 \mathrm{~nm}$ to $544 \mathrm{~nm}$ as shown in Figure 1. The formations of phase change and crystalline nature of $\mathrm{Au}$ nanoparticles were confirmed by $\mathrm{X}$-ray diffraction patterns of synthesized gold nanostructure produced by the leaf extract in support with UV-Vis analysis.

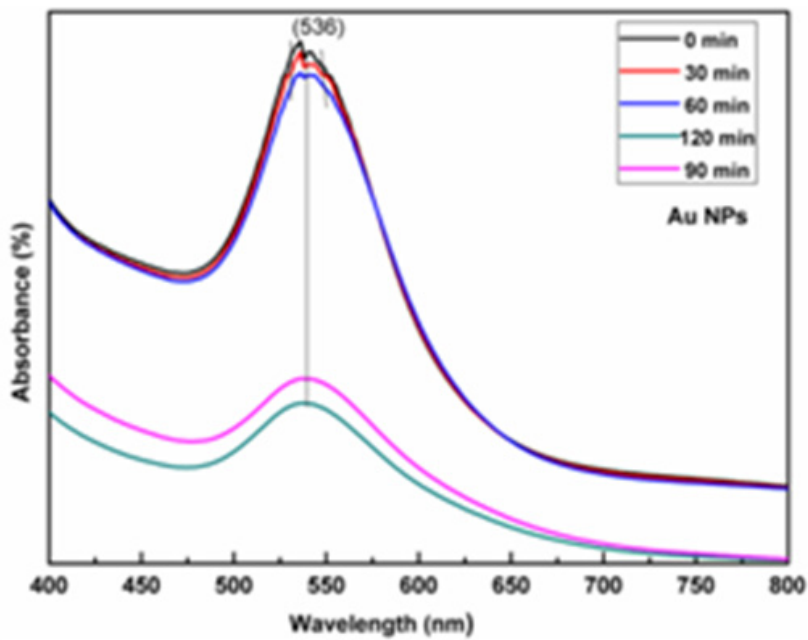

Figure I the UV-Vis absorption spectra of reduction of gold ions to AuNPs synthesized by Pergularia daemia leaf extract with different time intervals $0,30,60,90$ and 120 mins respectively.

\section{XRD Analysis}

Further the result of the UV-Vis spectra of the biosynthesized AuNPs were confirmed by the characteristic peaks observed in the XRD image and the structural view under the scanning electron microscope as shown in Figure 2. The formations of phase change and crystalline nature of Au nanoparticles were confirmed by X-ray diffraction patterns of synthesized gold nanostructure produced by the leaf extract in support with UV-Vis analysis. The characteristics peaks of $\mathrm{Au}$ nanoparticles are located at peak value of $2 \Theta=38.12^{\circ}, 44.4^{\circ}$, $65.1^{\circ}$ and $78.12^{\circ}$ for the indexing angles of reference planes at (111), (200), (220), and (311) as shown in Figure 1, which well matches with data base of JCPDS number : 04-0784. A very high crystalline and nano level particles with face-centered cubic (fcc) crystallographic references planes of Au nanoparticles. The average crystalline size of the nanoparticles formed in the bio reduction process found to be 32 $\mathrm{nm}$. The lattice constant calculated from this pattern was $4.0885 \mathrm{~A}$. The analysis revealed spherical structure of AuNPs and high peaks indicates the active silver composition and crystalline nature of the AuNPs. The compound is stable, which is confirmed by its angle value. The diffracted intensities were recorded from $2 \theta$ angles and values were used to calculate the grain size by using the Scherer's formula.

$$
D=\frac{(0.9 \lambda)}{\beta \cos \theta}
$$

Where, D-is the average crystallite domain size perpendicular to the reflection planes

$\lambda$-is the $\mathrm{X}$ - ray wavelength, $\beta$-is the full width at half maximum (FWHM) and $\Theta-$ is the diffraction angle. 


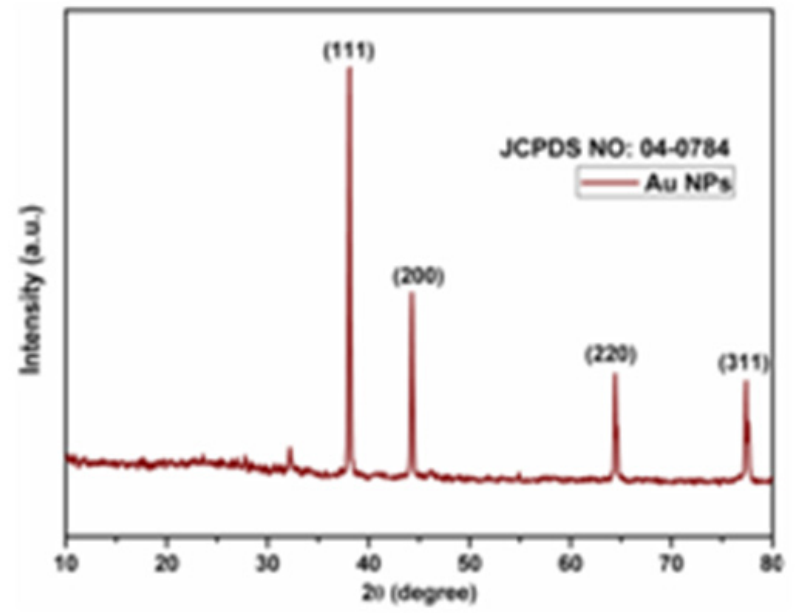

Figure 2 XRD patterns of AuNPs by treating Pergularia daemia leaf extract.

The average of grain size of the AuNPs formed in the bioreduction process was determined using Scherer's formula and was estimated at $32 \mathrm{~nm}$. The calculated lattice constant from this pattern was $4.085 \mathrm{~A} \square$. The XRD results clearly show that AuNPs was formed by the reduction $\mathrm{Au}+$ ions AuNPs and revealed the spherical structure of AuNPs and the high peaks indicates the active silver composition and crystalline nature of the AuNPs. The compound is stable, which is confirmed by its angle values.

\section{FESEM analysis}

The FESEM are the best analysing tool for structural and morphological properties of synthesised materials. Figure 3 shows structure and the morphology of AuNPs investigated using FESEM analysis. The images reveals that the green synthesized AuNPs particles are spherical/sphere like structure with microporous nature in shape with agglomerations, and the dimensions of the spherical shape are between $45 \mathrm{~nm}$ in width of smooth surface of AuNPs which have well dispersive property. This image reveals that the green synthesized AuNPs exhibits that almost all the particles are spherical shape with agglomeration and the dimensions of spherical shape are between 50-200 $\mathrm{nm}$ in width observed in different magnification.
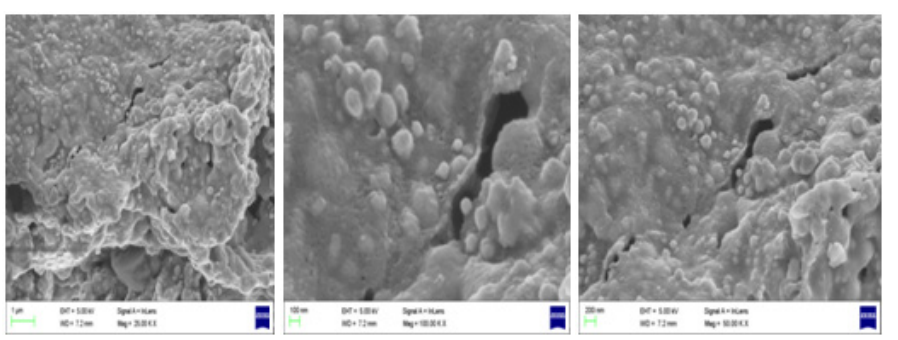

Figure 3 FESEM image of AuNPs at (a) I $\mu \mathrm{m}$ (b) $100 \mathrm{~nm}$ and (c) $200 \mathrm{~nm}$.

\section{HRTEM Analysis}

The morphology and internal crystalline structure of obtained nanoparticles was studied by the high resolution transmission electron microscopy and the selected area electron diffraction is carried out to find lattice planes of index (HRTEM and SAED) as shown in Figure 4. The HRTEM image of synthesized AuNPs was found to be spherical shaped grain like morphology with various radius ranging from 0.5 $\mathrm{nm}$ to $5 \mathrm{~nm}$. It was found that AuNPs spherical shaped with minimum particles sizes ranges $10 \mathrm{~nm}$ scales is shown in Figure 4. The selected area electron diffraction (SAED) patterns confirmed the presence of elementals AuNPs which well matches with the crystallographic data resulted of XRD analysis. The diffraction rings agrees with JCPDS data major indexing peaks (311) (Tabble 1).

Table I UV- Characterization of synthesized AuNPs at different time intervals

\begin{tabular}{lll}
\hline S.NO & Different Time & Surface Plasmon Resonance Peak (nm) \\
\hline I & 0 min & 544 \\
2 & $30 \mathrm{~min}$ & 542 \\
3 & $60 \mathrm{~min}$ & 540 \\
4 & $90 \mathrm{~min}$ & 538 \\
5 & $120 \mathrm{~min}$ & 536 \\
\hline
\end{tabular}
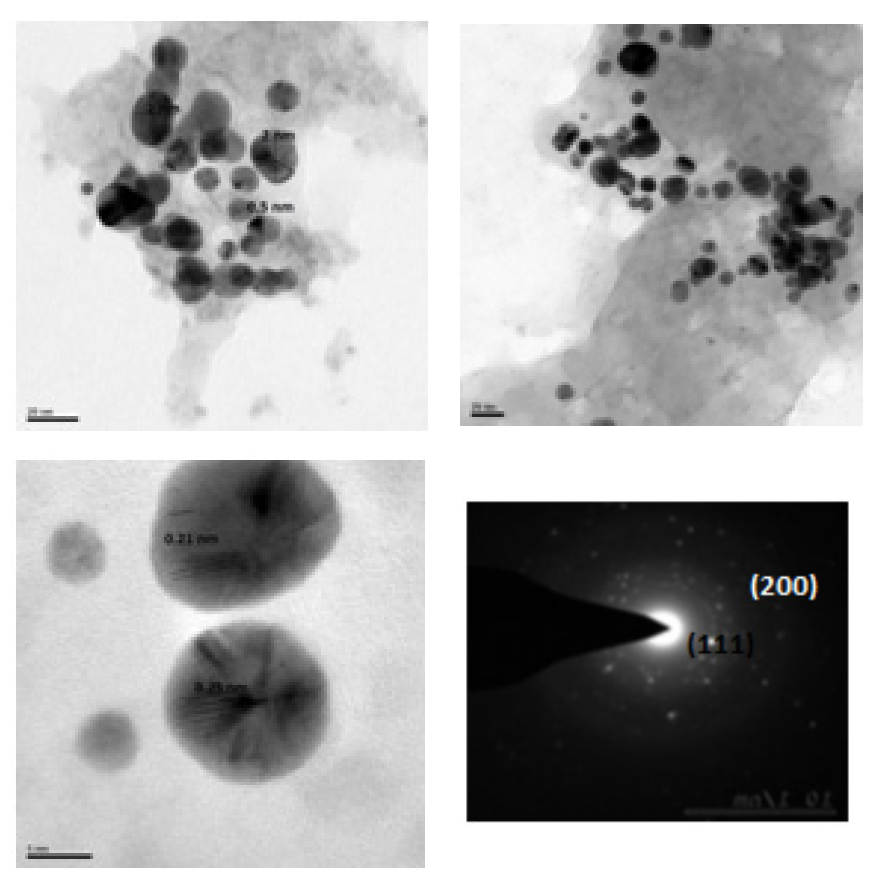

Figure 4 HRTEM images of synthesized AuNPs at different magnification of (a) $20 \mathrm{~nm}$, (b) $20 \mathrm{~nm}$ (c) $5 \mathrm{~nm}$ and (d) SAED patterns.

\section{Antimicrobial Activity}

Gold ions as well as AuNPs were known to have strong antimicrobial activities. The antibacterial activity of different concentration of solutions containing AuNPs demonstrated that both gram positive and gram negative bacteria were inhibited. AuNPs exhibited antimicrobial activity against multi-drug resistant (MDR) human pathogenic gram negative gram-negative bacteria like E.coli, Pseudomonas aeruginosa and gram-positive bacteria like Bacillus subtilis respectively. The inhibition rate at concentration of $100 \mu \mathrm{L}$ (A1), $200 \mu \mathrm{L}$ (A2), $300 \mu \mathrm{L}(\mathrm{A} 3$ ) and AuNPs the surface of bacteria cell membrane that disturbing the permeability and respiration functions as shown (Figure 5a-5c). The results of the antibacterial assay are depicted in Figure 5. The activity of these solutions was mainly due to the different amounts of AuNPs formed upon addition of different concentrations of Pergularia daemia. The result shows that the zone of inhibition (ZOI) was increased of AuNPs.

The maximum antibacterial activity in $300 \mu \mathrm{L} / \mathrm{mL}$ concentrations of the synthesized Au NPs was $19 \mathrm{~mm}, 17 \mathrm{~mm}$ and $16 \mathrm{~mm}$ for gram-negative bacteria like E.coli, Pseudomonas aeruginosa and gram-positive bacteria like Bacillus subtilis respectively. E. coli shows highest antibacterial activity for synthesized AuNPs by Pergularia daemia leaf extract than other bacteria. This confirms that AuNPs from Pergularia daemia exhibited good antibacterial potential against gram-negative bacterial strains when compared to gram positive strain. 

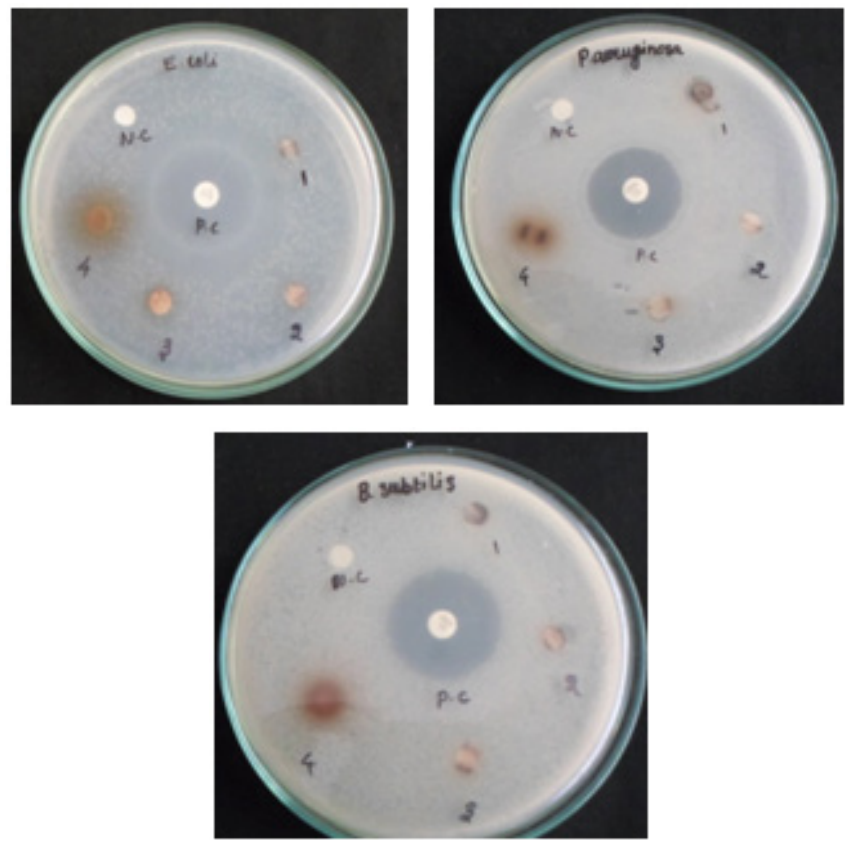

Figure 5 Antibacterial activity of synthesized gold nanoparticles (leaf extract) on (a) gram-negative bacteria like E.coli, Pseudomonas aeruginosa and grampositive bacteria like Bacillus subtilis respectively by the disk diffusion method.

\section{Conclusion}

In conclusion a simple, cost-effective and green synthesis of AuNPs by using Pergularia Daemia leaf extract as reducing agents, which requires no special physical conditions and can potentially replace and eliminate the problem of chemical agents that have may adverse effects, thus making nanoparticles more biocompatible with the eco-friendly approach. AuNPs have potential stability over the antibacterial activities against gram-negative bacteria like $E$. coli, Pseudomonas aeruginosa and gram-positive bacteria like Bacillus subtilis respectively which are dependent on the concentration of $\mathrm{Au}$ ionic nature. The formation of AuNPs was confirmed by UV-visible spectroscopy and XRD. The transmission electron microscopic images showed that the particles were of mostly spherical shape with the average particles size of ranging from $3 \mathrm{~nm}$ to $15 \mathrm{~nm}$. The $p H$, incubation condition and temperature conditions did not affect the growth of AuNPs treated with cells reveals that green synthesized AuNps shows an active and stable antibacterial activity in both grampositive and gram-negative bacteria, but it is more effective for gramnegative organisms when compared with gram-positive organisms. As because of its significant antibacterial efficacy, it may have potential applications in the field of biomedicine. Finally, it can be extendable to large scale production of Au NPs for commercial use.

\section{Conflicts of Interest}

The authors declare no conflict of interest.

\section{Acknowledgments}

None.

\section{References}

1. Sulaiman GM, Mohammed WH, Marzoog TR et al. Green synthesis antimicrobial and cytotoxic effects of Silver nanoparticles using Eucalyptus chapmaniana leaves extract. Asian Pac J Trop Biomed. 2013;3(1):58-63.
2. Thirumurugan A, Neethu, Anns Kumar prakash p Formulation and In Vitro Evaluation of Stavdine loaded human serium albumin nanoparticles. International journal of Nonmaterial's and Biostructures. 2011;373:139.

3. Sivakumar J, Premkumar C, Santhanam Biosynthesis of silver nanoparticles Using Calotropis gigantean leaf. African journal of basic applied sciences. 2014;3(6):265-270.

4. Akal M Awwad, Nida M Salem, Amany O Abdeen Green synthesis of silver nanoparticles using carob leaf extract and its trial antibacterial activity. International journal of Industrial chemistry. 2013;4(1):29.

5. John Xavier R, Arumugam M, R Bhuvaneswari Bio-Fabrication, Characterization of Silver Nanoparticles and their Evaluation of Catalytic, Antioxidant and Antimicrobial Efficacy. IOSR Journal of Applied Physics (IOSR-JAP). 2015;7(3):P. 76-81.

6. Devi Priyanka, Annamalai, PTV Lakshmi Reduction of Silver Ions by Cell Free Extracts of Westiellopsis sp. International Journal of Biomaterials. 2015;(2015): 6

7. Moreno Garrido I, Pérez S, Blasco J Toxicity of silver and gold nanoparticles on marine microalgae. Mar Environ Res. 2015;111:60-73.

8. Goodsell DS Bionanotechnology: lessons from nature. Hoboken, USA. 2004

9. Templeton AC, Wuelfing WP, Murray RW Monolayer protected cluster molecules. Acc Chem Res. 2000;33(1):27-36.

10. Morones JR, Elechiguerra JL, Camacho A et al. The bactericidal effect of silver nanoparticles. Nanotechnology. 2005;16(10):2346-2353.

11. Sambhy V, MacBride MM, Peterson BR, Sen A Silver bromide nanoparticle/polymer composites: dual action tunable antimicrobial materials. J Am Chem Soc. 2006;128(30):9798-9808.

12. Raveendran P, Fu J, Wallen SLA simple and green method for the synthesis of $\mathrm{Au}, \mathrm{Ag}$, and $\mathrm{Au}-\mathrm{Ag}$ alloy nanoparticles. Green Chem. 2006;8:34-38.

13. Saxena A, Tripathi RM, Singh RP Biological synthesis of silver nanoparticles by using Onion (Allium cepa) extract and their antibacterial activity. Digest Journal of Nanomaterials and Biostructres. 2010;5(2):427-432.

14. Magudapathy P, Gangopadhyay P, Panigrahi B K, Nair KGM, Dhara S Synthesis of Silver nanoparticles with antibacterial activity using the lichen parmotrema praesorediosum. Physica. 2001;1(2):142-146.

15. Xia L, Lenaghan SC, Zhang M, Zhang Z, Li Q Naturally occurring nanoparticles from English Ivy: An alternative to metal based nanoparticles for UV protection. Journal of Nanobiotechnology. 2010;8:12.

16. Ying Li, Shen Ming Chen, M Ajmal Ali, Fahad M A, AlHemaid Biosynthesis and Electrochemical Characterization of Silver Nanoparticles from Leaf Extract of Adeniumobesum and Its Application to Antibacterial Effect. Int J Electrochem Sci. 2013;8(1):2691-2701.

17. Sulaiman GM, Mohammed WH, Marzoog TR et al. Green synthesis, antimicrobial and cytotoxic effects of silver nanoparticles using Eucalyptus chapmaniana leaves extract. Asian Pac J Trop Biomed. 2013;3(1):58-63.

18. Sangeethaarun, Saraswathi U, Singaravelu Green synthesis of silver nanoparticles using a mangrove Excoecaria agallocha. International Journal of Pharmaceutical Science Invention. 2014;3(10): p. 54-57.

19. Ajitha B, Ashok Kumar Reddy Y, Sreedhara Reddy P Green synthesis and characterization of silver nanoparticles using Lantana camara leaf extract. Mater Sci Eng C Mater Biol Appl. 2015;49:373-381.

20. Gajbhiye M, Kesharwani J, Ingle A et al. ungus-mediated synthesis of silver nanoparticles and their activity against pathogenic fungi in combination with fluconazole. Nanomedicine. 2009;5(4):382-386. 